\title{
Philosophiques
}

\section{Un souffle sur la nuque : quand la perception devient affective}

\section{Frédérique de Vignemont}

Volume 45, numéro 2, automne 2018

URI : https://id.erudit.org/iderudit/1055273ar

DOI : https://doi.org/10.7202/1055273ar

Aller au sommaire du numéro

Éditeur(s)

Société de philosophie du Québec

ISSN

0316-2923 (imprimé)

1492-1391 (numérique)

Découvrir la revue

Citer ce document

de Vignemont, F. (2018). Un souffle sur la nuque : quand la perception devient affective. Philosophiques, 45(2), 467-476. https://doi.org/10.7202/1055273ar d'utilisation que vous pouvez consulter en ligne.

https://apropos.erudit.org/fr/usagers/politique-dutilisation/ 


\title{
Un souffle sur la nuque: quand la perception devient affective
}

\author{
FRÉDÉRIQUE DE VIGNEMONT \\ Institut Jean Nicod, Ecole Normale Supérieure, PSL, Paris, France \\ frederique.de.vignemont@ens.fr
}

\section{Un modèle perceptif des émotions?}

Le livre de Christine Tappolet, Emotions, values and agency, est l'occasion pour elle non seulement d'offrir un cadre théorique cohérent et extrêmement riche pour mieux appréhender les émotions dans leur rapport à la perception, l'action, les valeurs et la responsabilité, mais aussi de répondre à un certain nombre de critiques qui lui ont été faites par le passé, renforçant ainsi le caractère convaincant de sa théorie. La clarté du propos et la rigueur conceptuelle rendent la lecture de l'ouvrage tout à fait stimulante, même pour une non-experte des émotions. Sa théorie évaluative apparaît comme un compromis très intéressant entre deux positions extrêmes sur les émotions, la sur-incarnation d'une théorie à la William James et la surintellectualisation d'une théorie cognitive. Elle évite en effet les écueils sur lesquels s'échouent ces deux options. Elle permet en particulier de tenir compte de l'objet intentionnel des émotions. De la même manière que les expériences sensorielles, les émotions ont un contenu représentationnel. Elles ne portent pas seulement sur l'état du corps, mais sur le chien qui fait peur ou le cadeau qui rend heureux. Pour autant, ce contenu intentionnel n'exige pas de ressources cognitives telles que les jeunes enfants et les animaux ne pourraient pas en avoir l'expérience. Il n'implique pas d'émettre un jugement évaluatif, mais seulement de représenter de manière non conceptuelle la valeur de l'objet. De même que j'ai une conscience primitive et immédiate de la couleur bleue du ciel, j'ai conscience du chien comme étant effrayant, et cette expérience évaluative constitue mon émotion de peur. Pour Tappolet en effet, les émotions sont comparables aux expériences sensorielles et constituent une forme de perception. Comparons ainsi l'expérience visuelle qui résulte de l'illusion de Muller-Lyer (je vois une ligne plus grande que l'autre alors même qu'elles sont de taille identique) et le sentiment de peur face au vide. Il existe un certain nombre d'aspects sur lesquels émotion et perception sensorielle se rejoignent, comme le résume le tableau suivant. 
- Philosophiques / Automne 2018

\begin{tabular}{|c|c|c|}
\hline & Expérience visuelle & Expérience de peur \\
\hline Phénoménologie & $\begin{array}{l}\text { Cela me fait un certain effet } \\
\text { de voir les lignes. }\end{array}$ & $\begin{array}{l}\text { Cela me fait un certain effet } \\
\text { d'avoir peur. }\end{array}$ \\
\hline Dimension passive & $\begin{array}{l}\text { Je ne peux décider de les } \\
\text { voir comme étant verticales } \\
\text { plutôt qu'horizontales. }\end{array}$ & $\begin{array}{l}\text { Je ne peux décider d'être } \\
\text { excitée plutôt que d'avoir } \\
\text { peur. }\end{array}$ \\
\hline Réponse au monde & $\begin{array}{l}\text { Mon expérience est causée } \\
\text { par les lignes présentées } \\
\text { devant moi. }\end{array}$ & $\begin{array}{l}\text { Mon expérience est causée } \\
\text { par le vide devant moi. }\end{array}$ \\
\hline $\begin{array}{l}\text { Conditions } \\
\text { de correction }\end{array}$ & $\begin{array}{l}\text { Je suis dans l'erreur si les } \\
\text { lignes sont identiques. }\end{array}$ & $\begin{array}{l}\text { Je suis dans l'erreur si je ne } \\
\text { risque rien. }\end{array}$ \\
\hline $\begin{array}{l}\text { Encapsulation } \\
\text { informationnelle }\end{array}$ & $\begin{array}{l}\text { Je peux faire l'expérience } \\
\text { de l'illusion alors même que } \\
\text { je sais que les lignes ont } \\
\text { la même taille. }\end{array}$ & $\begin{array}{l}\text { Je peux avoir peur alors que } \\
\text { je sais que je ne peux pas } \\
\text { tomber. }\end{array}$ \\
\hline $\begin{array}{l}\text { Isolement } \\
\text { inférentiel }\end{array}$ & $\begin{array}{l}\text { Mon expérience des lignes } \\
\text { n'est ni inférée de mes } \\
\text { croyances ni mes croyances } \\
\text { inférées de mon expérience } \\
\text { visuelle. }\end{array}$ & $\begin{array}{l}\text { Ma peur n'est ni inférée } \\
\text { de mes croyances ni mes } \\
\text { croyances inférées de } \\
\text { ma peur. }\end{array}$ \\
\hline $\begin{array}{l}\text { Contenu } \\
\text { analogique }\end{array}$ & $\begin{array}{l}\text { Mon expérience des lignes } \\
\text { se modifie subtilement au } \\
\text { fur et à mesure que leur } \\
\text { taille change. }\end{array}$ & $\begin{array}{l}\text { Mon expérience de peur se } \\
\text { modifie subtilement au fur } \\
\text { et à mesure que le vide sous } \\
\text { mes pieds s'agrandit. }\end{array}$ \\
\hline
\end{tabular}

Tappolet reconnaît, malgré ces similarités, l'existence d'un certain nombre de différences. La phénoménologie affective, en particulier, est plus complexe. À la différence des expériences sensorielles, l'effet que cela fait d'avoir peur peut se décrire à de multiples niveaux, du niveau intéroceptif le plus bas au niveau cognitif et conatif le plus haut. En outre, les émotions se caractérisent par un sentiment hédonique: elles sont plaisantes ou déplaisantes. De plus, elles jouent un rôle motivationnel. À la différence des expériences sensorielles, les émotions sont intimement liées à l'action. D'autre part, toujours à la différence des expériences sensorielles, les émotions ne sont pas en contact direct avec le monde, mais reposent plutôt sur une base cognitive constituée de ce que l'on perçoit, imagine, croit, et désire. Par conséquent, l'émotion ressentie dans un certain contexte peut être en partie déterminée par des facteurs socio-culturels. À la différence de ce que nous vivons dans le cas des expériences sensorielles, il est donc possible de demander pourquoi nous ressentons telle ou telle émotion, et ce que nous 
éprouvons peut être irrationnel. En outre, comme les émotions ne donnent pas un accès immédiat au monde, elles ne sont pas transparentes. Ainsi, nous avons conscience des émotions elles-mêmes, et pas uniquement de leur objet intentionnel. Une dernière différence notable est que, si l'on accepte la théorie de Tappolet, les émotions nous informent sur des propriétés normatives, et non sur des propriétés telles que la forme ou la couleur.

Devant un tel bilan, on peut s'interroger sur la dimension réellement perceptive des émotions. Les émotions consistent-elles en des expériences perceptives ou sont-elles seulement similaires à celles-ci? Et que faut-il entendre par perception? Il n'est pas toujours évident de savoir si la notion de perception joue un rôle clé ou non dans la théorie de Tappolet. D'un côté, elle insiste sur les similitudes avec les expériences sensorielles et cherche à réduire l'importance de leurs différences avec les émotions. D'un autre côté, elle comprend la notion de perception au sens large, comme ouverture au monde. Elle est même prête à concéder à ses opposants que ce qu'elle décrit peut n'être que de la «quasi-perception ». Pour elle, il ne s'agit là que d'un débat terminologique. On pourrait ainsi se demander si elle ne pourrait pas se contenter de parler d'une appréhension non conceptuelle de valeurs. Elle éviterait alors un certain nombre de critiques qu'on a pu lui faire (Brady, 20I3). Mais doit-elle abandonner aussi vite le modèle perceptif? Un regret que l'on peut avoir en lisant son chapitre I est paradoxalement le peu d'attention porté aux expériences sensorielles. Tappolet prend en effet pour point de départ une conception très classique, et potentiellement datée, du fonctionnement de la vision, négligeant ainsi un certain nombre de débats actuels. Pour ne prendre que quelques exemples, l'hypothèse fodorienne d'encapsulation d'informations des modules sensoriels a été remise en cause ces dernières années à la suite d'une série d'expériences montrant l'influence des croyances sur les expériences visuelles (voir Macpherson 20I2, pour discussion). Depuis quelques années émerge aussi une nouvelle conception de la perception formulée en termes d'encodage prédictif, selon laquelle nos expériences sensorielles sont loin de nous donner un accès direct au monde. Au contraire, elles s'appuieraient sur une base cognitive, constituée par l'anticipation de ce que nous allons percevoir, formée sur la base de nos connaissances antérieures (Clark, 20I3; Hohwy, 20I3). Plus récemment encore, Susanna Siegel (20I6) a défendu l'hypothèse selon laquelle la perception sensorielle obéit elle aussi aux lois de la rationalité, et que nos expériences visuelles ont une charge épistémique.

Je ne m'attarderai ici sur aucune de ces nouvelles tendances, qui, si elles étaient confirmées, renforceraient le parallèle entre les expériences sensorielles et les émotions. Je me focaliserai simplement sur la question du lien avec l'action, question qui joue un rôle central dans le livre, et plus particulièrement dans le chapitre III. Lorsque je vois un chien menaçant, ma peur me conduit à reculer doucement et à m'éloigner le plus possible. Elle me pousse à agir et justifie mon comportement. La situation apparaît totale- 
ment différente dans le cas des expériences sensorielles. La vision de deux lignes parallèles me laisse froide, si je puis dire. Elle est dénuée de force motivationnelle. Si j'agis, c'est à la seule condition que je forme le désir de le faire. Face à cette différence, Tappolet cherche à montrer que le rapport à l'action n'est pas toujours aussi direct et évident dans le cas des émotions. Comme elle le décrit très bien, nous avons de nombreuses peurs qui ne provoquent aucun comportement spécifique. Par exemple, nous craignons l'énergie nucléaire ou une crise boursière sans pour autant que notre comportement s'en ressente. Nous pouvons aussi avoir ce qu'elle appelle des sentiments contemplatifs dans des œuvres de fiction, qui sont eux aussi dissociés de toute action. Ainsi, les émotions ne sont pas aussi différentes des expériences sensorielles qu'on le suppose. Ma stratégie ici sera l'inverse de la sienne: je vais montrer que les expériences sensorielles ne sont pas toujours aussi différentes des émotions que même Christine Tappolet le suppose. Nous allons voir que l'espace qui entoure notre corps, dit espace péripersonnel, est perçu de manière particulière, et que les expériences visuelles de cet environnement à portée de main ont une force motivationnelle en partie similaire à celle des émotions. Nous verrons en outre qu'une manière d'en rendre compte a trait au contenu évaluatif. Nous verrons ensuite quelles implications une telle interprétation a pour la théorie perceptive de Tappolet.

\section{La perception péripersonnelle}

Beaucoup de choses dans le monde m'importent, mais seules quelques-unes ont une signification immédiate. La notion d'immédiateté doit être comprise ici à la fois spatialement et temporellement. Tout ce qui se passe dans mon espace péripersonnel peut avoir des implications directes sur mon bien-être et sur ce que je ferai dans un proche avenir. L'espace péripersonnel est l'espace dans lequel le monde est littéralement à portée de main pour interagir avec lui. C'est aussi l'espace dans lequel le monde peut devenir menaçant et dangereux, exigeant des comportements de défense. Il n'est donc pas étonnant que la perception de cet espace (ce que j'appelle la perception péripersonnelle) présente un certain nombre de caractéristiques distinctes de la perception de l'espace plus lointain (pour une revue empirique détaillée, voir Brozzoli et al., 20 I2).

C'est le directeur du zoo de Zurich, Heini Hediger (I950), qui le premier a décrit que l'animal réagit de manière spécifique lorsqu'il perçoit qu'un prédateur s'approche de trop près. Il y aurait ainsi autour de l'animal une marge de sécurité dont l'invasion provoque automatiquement un comportement de défense. En I98I, Rizzolatti et ses collègues nomment cette zone l'espace péripersonnel. Ils montrent que chez le singe certains neurones dans les aires prémotrices s'activent non seulement lorsque l'animal est touché, mais aussi lorsqu'il voit un stimulus visuel proche de son corps (jusqu'à $30 \mathrm{~cm}$ ). De même, chez l'humain, on s'est aperçu que la perception 
visuelle d'un flash interfère avec une perception tactile simultanée, mais seulement si le flash apparaît dans l'espace péripersonnel (Spence et al., 2004). Cet effet s'explique par le fait que ce qui est dans l'espace péripersonnel risque grandement d'être très bientôt en contact avec le sujet, et le système sensoriel ne fait qu'anticiper ce futur contact afin d'y être mieux préparé. C'est pourquoi le référentiel spatial des expériences péripersonnelles est somatotopique: les stimuli externes sont localisés par rapport aux parties du corps qui risquent d'être en contact avec eux.

Une autre particularité de la perception péripersonnelle est que les objets sont plus vite détectés quand ils sont proches (Reed et al., 2010). L'hypothèse est que l'espace péripersonnel a automatiquement priorité pour les ressources attentionnelles. Le fait est qu'on ne peut se permettre d'attendre pour traiter les informations, car ce qui est proche a des implications immédiates. La rapidité, toutefois, n'implique pas que le système bâcle son travail. Au contraire, il a été montré dans une tâche de recherche visuelle que les participants mettent plus longtemps à localiser une cible au milieu de distracteurs dans l'espace péripersonnel (Abrams et al., 2008). De nouveau, cela s'explique facilement. Imaginez que vous êtes dans la forêt amazonienne. Vous pouvez vous permettre de ne pas avoir une connaissance approfondie de ce qui se trouve au loin, mais vous avez besoin de scruter de manière systématique toutes les feuilles et lianes à côté de vos pieds pour être sûr qu'un serpent ne s'y cache pas. Enfin, le traitement des informations sur la forme globale et le mouvement est facilité dans l'espace péripersonnel, mais pas les informations sur la couleur et les détails spatiaux (Goodhew et al., 20I5). Ce qui compte, c'est de pouvoir identifier qu'il y a une menace potentielle afin de réagir, et non d'en connaître tous les détails.

Les spécificités de la perception péripersonnelle s'expliquent ainsi par son lien à l'action, et plus précisément à la protection de soi pour laquelle elle s'est développée. La fonction de la perception péripersonnelle est en priorité défensive, et plus généralement motrice (Rizzolatti et al., 1997; Brozzoli et al., 20I2; Graziano, 2009). Le neuroscientifique Graziano et ses collègues ont ainsi découvert: (i) que la perception des stimuli proches du corps provoque un comportement d'évitement, et (ii) que la stimulation électrique directe des neurones bimodaux impliqués dans la perception péripersonnelle suscite également des réponses protectrices. Il a été démontré, chez l'homme aussi, que la perception d'un son intense près de la main peut provoquer une réaction d'inhibition de type défensif, ressemblant à celle observée lors de la présentation de stimuli nociceptifs (Avenanti et al., 20I 2). L'espace péripersonnel est littéralement l'espace "à l'intérieur duquel [le corps] peut agir» (Maravita et al., 2003, page 53 I). Le guidage de l'action dépend de sa surveillance constante. Il n'est donc pas surprenant qu'au niveau cérébral l'espace péripersonnel soit représenté dans les aires corticales impliquées dans le contrôle de l'action. Ainsi, pour résumer, la perception péripersonnelle semble respecter le vieil adage scout "Toujours prêt", 
car ce qui est proche de nous est ce pour quoi nous devons être préparés à réagir sans attendre l'intervention d'états conatifs additionnels.

\section{Un modèle affectif de la perception?}

Ce que les résultats sur l'espace péripersonnel révèlent, c'est la possibilité que la vision puisse entretenir un lien causal direct avec l'action. Cette idée existe depuis longtemps, que ce soit chez le psychologue gestaltiste Kurt Koffka (I935) ou plus tard chez le psychologue écologiste James Gibson (1969) (voir Siegel, 20I4 pour une récente discussion). Koffka prend l'exemple suivant: si vous avez un courrier à envoyer, l'expérience visuelle d'une boîte aux lettres exerce une force d'attraction sur vous qui vous conduit à poster votre lettre. Ce que les récentes découvertes sur l'espace péripersonnel apportent par rapport aux hypothèses de Koffka et de Gibson, ce sont les conditions à remplir pour que cette force s'exerce: la boîte aux lettres doit être juste à côté de vous, et non de l'autre côté de la rue, pour que le lien entre l'expérience visuelle et l'action soit direct (pour une plus ample discussion, voir de Vignemont, 2018). La relation perception-action peut apparaître alors comme étant similaire à celle que l'on retrouve dans les émotions. Cela semble tout particulièrement frappant dans le cas de la perception de menace dans l'espace péripersonnel: nous pouvons réagir automatiquement comme lorsque nous avons peur.

Mais on pourrait répondre que c'est précisément parce que nous avons peur que nous pouvons nous conduire ainsi. Autrement dit, voir une menace proche provoque un sentiment de peur, qui peut provoquer à son tour un comportement de défense. Selon cette interprétation, le contenu sensoriel reste standard, si l'on peut dire, sans force motivationnelle. Si tel était le cas, alors la perception sensorielle, même de l'espace péripersonnel, resterait toujours différente des émotions. Cette interprétation toutefois ne semble pas se justifier. Il ne s'agit pas de nier que la peur joue un rôle, mais de savoir si elle est la seule à nous pousser à agir, ou s'il est possible que l'expérience visuelle ait une influence immédiate sur notre comportement. On peut se demander par exemple, si un prédateur apparaît subitement dans l'espace proche, si nous avons véritablement le temps d'avoir peur, et ensuite seulement d'agir. En outre, si on vous demande pourquoi vous retirez votre main vers laquelle volait une abeille, vous répondrez probablement qu'elle était trop proche de vous. Vous n'avez pas nécessairement peur de l'abeille en tant que telle. Le contenu visuel justifie votre comportement: il rend intelligible la raison pour laquelle vous réagissez. Il n'est même pas besoin de faire appel à de méchantes petites bestioles pour illustrer le rôle motivationnel de la perception péripersonnelle. Il suffit de naviguer dans une pièce pleine de meubles. Au fur et à mesure que nous avançons, nous percevons ces objets comme entrant dans un espace péripersonnel et nous les contournons automatiquement. Très clairement, nous n'avons pas peur de la chaise ou de la table. Et pourtant nous les évitons. Il semble donc qu'on ne puisse réduire 
les spécificités de la perception péripersonnelle à la simple combinaison d'un état sensoriel standard et d'une émotion. Il paraît plus plausible de soutenir que le contenu sensoriel est similaire au contenu émotionnel.

Pour mieux comprendre ce modèle affectif de la perception péripersonnelle, nous pouvons faire un détour par la sensation de douleur. Il a en effet été proposé de comprendre la douleur sur un modèle évaluatif. Par exemple, Bain (2013) la définit comme suit:

I) Une expérience somatosensorielle qui représente une perturbation spécifique du corps;

2) Cette expérience représente la perturbation comme étant mauvaise pour le corps.

On peut alors proposer une interprétation similaire des expériences péripersonnelles, qui consisteraient en:

I) Une expérience visuelle ou auditive qui représente une perturbation spécifique à proximité du corps;

2) Cette expérience représente la perturbation comme étant mauvaise pour le corps.

Le cadre conceptuel évaluativiste pourrait offrir un continuum qui va de la peur que vous ressentez quand vous voyez le serpent au loin, à l'expérience péripersonnelle que vous avez quand vous voyez le serpent approcher votre pied, pour finir par la douleur que vous ressentez quand le serpent vous a piqué 1 .

Cette continuité semble se confirmer sur le plan physiologique. De même que la peur et la douleur, la perception de menace dans l'espace péripersonnnel a une signature intéroceptive (comme l'augmentation du rythme cardiaque et de la conductance électrodermale). Les bases neurales sont aussi en partie les mêmes. Plus particulièrement, il a été trouvé qu'une large portion de ce que l'on appelle la matrice de la douleur (pain matrix) dans le cerveau est activée par la vision ou l'audition de stimuli saillants proches du corps (Legrain et al., 20I I). Enfin, d'un point de vue neuropsychologique, il est intéressant de noter que les patients qui souffrent d'asymbolie à la douleur ne réagissent ni aux stimuli douloureux ni aux menaces dans leur environnement proche (Grahek, 200I). En outre, une patiente ayant eu une double lésion de l'amygdale et qui n'éprouve plus de sentiment de peur montre aussi une indifférence totale quand un individu pénètre son espace péripersonnel, au lieu de se sentir de plus en plus inconfortable au fur et à mesure que la personne s'approche (Kennedy et al., 2009).

1. Il faut noter ici que la théorie évaluativiste de Bain présente certaines différences avec celle de Tappolet. En outre, ce continuum peut se retrouver dans d'autres conceptions, par exemple en termes de contenu à la fois descriptif et directif (Martinez, 20 I i pour la douleur; Scarantino, 20I4 pour les émotions). 
La continuité entre la peur, l'expérience de menaces proches et la douleur ne prouve pas la validité du modèle évaluativiste, et les critiques qui ont été émises à son encontre s'appliquent uniformément aux trois types d'expérience. $\mathrm{Si}$, par exemple, nous ne pouvons avoir un accès direct aux valeurs, comme le défendent Dokic et Lemaire (20I3), le contenu péripersonnel ne peut être du type évaluatif. Il n'en reste pas moins que cette continuité renforce l'argument par analogie qu'effectue Tappolet. Qu'importe le cadre théorique que l'on adopte pour expliquer les émotions, il doit prendre en considération le fait qu'elles partagent de nombreux points communs avec certaines expériences sensorielles, encore plus que ne le soupçonne Tappolet elle-même.

Mais on peut se demander si cette analogie renforcée ne risque pas de se retourner contre elle. Car il reste une différence fondamentale entre d'un côté la peur et la douleur, et de l'autre, l'expérience de menace proche: leur phénoménologie apparaît comme relativement distincte. La question devient alors pourquoi la peur nous fait-elle un effet si différent de l'expérience visuelle d'une menace si elles ont tant en commun? En particulier, dans le cadre d'une théorie intentionnaliste des qualia, si elles ont toutes deux un contenu évaluatif, elles devraient avoir la même phénoménologie. Trop de similarités avec les expériences sensorielles finiraient donc par nuire à la théorie perceptive des émotions. Néanmoins plusieurs voies sont ouvertes à Christine Tappolet. Une première réponse consisterait à minimiser la différence phénoménologique. Il se peut que voir un serpent à côté de son pied soit une sensation de tension désagréable et que les expériences péripersonnelles de ce type aient une qualité hédonique négative comme la douleur et la peur, même si elle est moins intense. Une autre solution serait d'accepter la différence phénoménologique et de l'expliquer par le mode de présentation du contenu. De même que voir et toucher un cube font un effet différent alors que les deux types d'expériences ont le même contenu, voir une menace et en avoir peur font aussi un effet différent. Enfin, il est possible d'expliquer la différence phénoménologique sur le plan du contenu lui-même. Dans son chapitre III, Christine Tappolet discute des différents types de valeurs susceptibles de faire partie du contenu de l'expérience de peur. Elle défend l'hypothèse que les valeurs émotionnelles sont des propriétés subjectives, qui dépendent de la réaction du sujet (response-dependent). Avoir peur du serpent, c'est avoir une expérience perceptive de son caractère effrayant. On pourrait proposer que, lorsque je ne fais que voir le serpent à côté de moi sans en avoir peur, je n'ai que l'expérience perceptive de son caractère dangereux. La valeur est alors objective au sens où elle ne dépend pas de la réaction du sujet, mais seulement des propriétés du monde.

En conclusion, la théorie de Christine Tappolet ouvre un large champ de questions sur la perception affective de manière plus générale. Je me suis concentrée ici sur le fait de voir une partie de son corps menacée, mais des enjeux similaires se retrouvent dans le cas de la perception esthétique. 


\section{Bibliographie}

Abrams, R. A., C. C. Davoli, F. Du, W. H. Knapp, \& D. Paull. «Altered Vision Near the Hands ", Cognition, 107 (3), 2008, I035-I047.

Avenanti, A., L. Annela, and A. Serino. "Suppression of Premotor Cortex Disrupts Motor Coding of Peripersonal Space», Neuroimage, 63 (I), 20I 2, 28 I-288.

Bain, D. «What Makes Pains Unpleasant? » Philosophical Studies, 2013, I66, S69S89.

Brady, M. Emotional Insight: The Epistemic Role of Emotional Experience, Oxford, Oxford University Press, 20I3.

Brozzoli, C., T. R. Makin, L. Cardinali, N. P. Holmes, and A. Farnè. « Peripersonal Space: a Multisensory Interface for Body-Object Interactions ", in M. M. Murray and M. T. Wallace (eds), The Neural Bases of Multisensory Processes, Boca Raton (FL), CRC Press, 2012.

Clark, A. "Whatever Next? Predictive Brains, Situated Agents, and the Future of Cognitive Science ", Behavioral and Brain Sciences, 36 (3), 20I3, I 8 I-204.

Dokic, J., \& S. Lemaire. "Are Emotions Perceptions of Value? ", Canadian Journal of Philosophy, 43 (2), 2013, 227-247.

Gibson, J. J. The Ecological Approach to Visual Perception, Boston, Boston Mifflin, I979.

Goodhew, S. C., M. Edwards, S. Ferber \& J. Pratt. «Altered Visual Perception Near the Hands: A Critical Review of Attentional and Neurophysiological Models, Neuroscience \& Biobehavioral Reviews, 55, 201 5, 223-233.

Grahek, N. Feeling Pain and Being in Pain, Cambridge, MA, MIT Press, $200 \mathrm{I}$.

Graziano, M. The Intelligent Movement Machine: An Ethological Perspective on the Primate Motor System, Oxford, Oxford University Press, 2009.

Hediger, H. Wild Animals in Captivity, London, Butterworths Scientific Publications, I950.

Hohwy, J. The Predictive Mind, Oxford, Oxford University Press, 2013.

Kennedy D. P., J. Gläscher, JM Tyszka, \& R. Adolphs. «Personal Space Regulation by the Human Amygdala", Nat Neurosci, I 2 (IO), 2009, I 226 -I 227.

Koffka, K. Principles of Gestalt Psychology, London, Kegan Paul Trench, Trubner \& Co, I935.

Legrain, V., G. D. Iannetti, L. Plaghki, \& A. Mouraux. «The Pain Matrix Reloaded: a Salience Detection System for the Body », Progress in Neurobiology, 93 (I), 2OII, III-I24.

Macpherson, F. "Cognitive Penetration of Colour Experience: Rethinking the Issue in Light of an Indirect Mechanism", Philosophy and Phenomenological Research, 84 (I), 2012, 24-62.

Maravita, A., C. Spence, and J. Driver. "Multisensory Integration and the Body Schema: Close to Hand and Within Reach, Current Biology, I3 (I3), 2003,

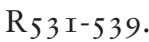

Martínez, M. "Imperative Content and the Painfulness of Pain ", Phenomenology and the Cognitive Sciences, 10 (I), 20I I, 67-90.

Reed, C. L., R. Betz, J. P. Garza \& R. J. Roberts. «Grab it! Biased Attention in Functional Hand and Tool Space", Attention, Perception, \& Psychophysics, 72 (I), 2010, 236-245. 


\section{Philosophiques / Automne 2018}

Rizzolatti, G., C. Scandolara, M. Matelli, and M. Gentilucci. «Afferent Properties of Periarcuate Neurons in Macaque Monkeys. II. Visual Responses », Behavioural Brain Research, 2 (2), I98 I, I47-I63.

Scarantino, A. "The Motivational Theory of Emotions", Moral Psychology and Human Agency, Justin D’Arms et Daniel Jacobson (dir.), 20I4, p. I 56-I 85.

Siegel, S. The Rationality of Perception, Oxford, Oxford University Press, 2017.

Siegel, S. "Affordances and the Contents of Perception", in Does Perception Have Content? B. Brogaard (dir.), Oxford University Press, 2014.

Spence, C., F. Pavani and J. Driver. "Spatial Constraints on Visual-Tactile CrossModal Distractor Congruency Effects ", Cognitive, Affective, and Behavioral Neuroscience, 4 (2), 2004, I48-I69.

Tappolet, C. Emotions, values and agency, Oxford, Oxford University Press, 2016. de Vignemont, F. «Peripersonal Perception in Action », Synthese. 2018. 